\title{
Elastic-plastic adhesive contact of non-Gaussian rough surfaces
}

\author{
PRASANTA SAHOO*1 and S MOHAMED ALI ${ }^{2}$ \\ ${ }^{1}$ Department of Mechanical Engineering, Jadavpur University, Kolkata 700032 \\ ${ }^{2}$ Department of Mechanical Engineering, Pondicherry Engineering College, \\ Pondicherry 605014 \\ e-mail:psahoo@vsnl.net
}

MS received 19 September 2007; revised 28 April 2008

\begin{abstract}
The paper describes an analysis of adhesion at the contact between nonGaussian rough surfaces using the Weibull distribution with skewness as the key parameter to characterize asymmetry. The analysis uses an improved elastic-plastic model of contact deformation that is based on accurate Finite Element Analysis (FEA) of an elastic-plastic single asperity contact. Large range of interference values is considered starting from fully elastic through elastic-plastic to fully plastic regime of contacting asperities. The well-established elastic and plastic adhesion indices are used to consider the different conditions that arise as a result of varying load and material parameters. The loading and unloading behaviour for different combinations of the adhesion indices and skewness values are obtained as functions of mean separation between the surfaces. Transitional values of adhesion indices and skewness at which the influence of surface forces becomes insignificant are found to depend on material and surface parameters. Comparison with studies using previous elastic-plastic model that was based on some arbitrary assumptions shows significant differences in loading behaviour.
\end{abstract}

Keywords. Adhesion; asymmetric roughness; elastic-plastic contact; non-Gaussian rough surfaces.

\section{Introduction}

When two surfaces come into contact, the adhesional and tribological action takes place at the peaks of the asperities where intimate contact occurs and in this regard researchers have broadly used model of (GW) Greenwood \& Williamson (1966) to analyse such contacts. This model was primarily developed for engineering surfaces with relatively large initial roughness value of a few $\mu \mathrm{m}$ and has been used extensively despite its shortcomings e.g. scale dependence of some of its parameters (Whitehouse \& Archard 1970; Thomas \& Sayles 1978). The fully elastic GW asperity model has been extended to include elastic-plastic contacts both in presence and absence of adhesion (Roy Chowdhury \& Ghosh 1994; Chang et al

*For correspondence 
$1987 ; 1988)$. In all these studies the asperity heights are assumed to follow a symmetric Gaussian probability distribution function. However, in many engineering applications surfaces typically have 'Gaussian type' distributions but not necessarily symmetric (Williamson 1968; Whitehouse 1994; Stout et al 1990). Typical engineering surfaces that fall under this category include surfaces from machining operations in microtribology, e.g. grinding and turning (Whitehouse 1994; Stout et al 1990) and in miniature systems like micro-electromechanical systems (MEMS), head-disc interface in magnetic storage, etc. where micro machining and polishing are the prime machining operations (Bhushan 1999). Most of the common machining processes produce surfaces with non-Gaussian distributions (Thomas 1982). Turning, shaping and electro-discharge machining produce a peaked surface with positive skewness. Grinding, milling, honing and abrasion processes produce grooved surfaces with negative skewness and high kurtosis values. Mechanical texturing of disks with free abrasives or abrasive cloth result in a surface with deep valleys with negative skewness. Disk surfaces produced by sputter texturing (Mirzamaani \& Doerner 1996) have mainly peaks on the disk surfaces, which are expected to have positive skewness. Laser texturing also produces non-Gaussian surfaces (Ranjan et al 1991; Baumgart et al 1995). In these cases, McCool $(1992,2000)$ introduced the Weibull probability density function to model the distribution of asperity heights. This is a two-parameter distribution, compared to the one-parameter Gaussian distribution, and with proper choice of parameters can approximate the symmetric Gaussian distribution, as well as other asymmetric distributions. The asymmetry is characterized by the dimensionless parameter called skewness. The second non-dimensional characteristic of the distribution that is used to designate its flatness is the kurtosis. Distributions that are flatter or 'peakier' than the Gaussian are referred to as platykurtic and leptokurtic, respectively. For a Gaussian distribution, the skewness is equal to zero and the kurtosis is equal to three.

The interest in the study of adhesion between solids arises due to its significant influence on friction and wear of contacting surfaces. A great deal of progress has been made in this aspect both experimentally and analytically. Some of the pioneering work includes that of Bowden \& Rowe (1956), which shows the importance of surface film and released elastic stresses in reducing adhesion. When the smooth and clean surfaces are brought together, a finite force is required to separate the two due to the physical attractive and repulsive forces that become effective within a short range of separation. Two competing models for predicting these adhesive forces exist. These are widely known as the JKR (Johnson et al 1971) and DMT (Derjaguin et al 1975) models. In either case, the force of adhesion for an elastic sphere of radius $R$ is given by $C \pi R \gamma$, where $C$ is a constant, which is 1.5 and 2 for JKR and DMT models respectively, and $\gamma$ the work of adhesion given by $\gamma_{1}+\gamma_{2}-\gamma_{12}, \gamma_{1}$ and $\gamma_{2}$ being the surface energies for the two surfaces and $\gamma_{12}$ their interfacial energy. However, it has since been shown that the two models are the limiting cases of a generalised solution (Muller et al 1980). The influence of surface energy during plastic loading and unloading has been demonstrated (Roy Chowdhury \& Pollock 1981) and it was shown that an extra surface force term, $2 \pi R \gamma$, acts in addition to the applied load during plastic loading. Experimental work on this aspect has largely been carried out in high vacuum $(0.1 \mu \mathrm{Pa})$ with a relatively sharp tip (radius of only a few microns or less) brought into contact with a clean and smooth surface under extremely light loads of $1-20 \mu \mathrm{N}$. With these experimental techniques the force of adhesion at a typical single-asperity contact was obtained.

An important conclusion from the great deal of studies on adhesion and deformation is that the adhesion of clean surfaces could be greatly diminished due to surface roughness. One pioneering work in this aspect, carried out by Fuller \& Tabor (1975), describes the effect of surface roughness in terms of an elastic adhesion index, $\theta=K \sigma^{3 / 2} R^{1 / 2} /(\gamma R)$. This index 
is merely the ratio of elastic force needed to push a sphere of radius $R$ to a depth $\sigma$ into an elastic solid of equivalent modulus of elasticity $E$ to the adhesive force experienced by the sphere. In the present notation, $K=\frac{4}{3} E, E$ being $\left[\frac{1-v_{1}^{2}}{E_{1}}+\frac{1-v_{2}^{2}}{E_{2}}\right]^{-1}$, where $E_{1}$ and $E_{2}$ are Young's moduli, $v_{1}$ and $v_{2}$ are Poisson's ratios of two surfaces. Johnson (1976) likewise introduced an adhesion index for plastic deformation assuming an exponential distribution of asperity heights. This may be defined as $\lambda=\pi^{2} R H^{4} \sigma /\left(18 K^{2} \gamma^{2}\right)$ where $H$ is the hardness of the softer material. It may be noted here that $\lambda$ defined here is $1 / 16^{\text {th }}$ of $\lambda$ defined by Johnson (1976). Limiting values of $\theta$ and $\lambda$ are usually quoted as 10 and $0 \cdot 125$ respectively, beyond which the effect of adhesion becomes insignificant indicating that the surfaces are sufficiently rough for the surface forces to be inoperative. No quantitative criterion can be given for surface cleanliness, but it is known that even a monolayer of oxide or atmospheric contamination can reduce the surface energy of the parent materials significantly.

The effect of surface roughness on adhesion at the contact of rough solids has also been studied analytically in great detail by Chang et al (1987) (CEB model) and Roy Chowdhury \& Ghosh (1994) (RG model) using Greenwood and Williamson's rough surface model. The CEB model was based on the DMT adhesion model while the RG model used the JKR adhesion model. In RG model, it is considered that some contacting asperities remain purely elastic and other purely plastic and the model overlook a wide intermediate range of interest where elastic-plastic contact prevails. In CEB model an attempt was made to bridge this gap. In this model each contacting asperity remains in elastic Hertzian contact until a critical interference is reached, above which volume conservation of the asperity tip is imposed and a uniform average contact pressure is assumed. This simplifying assumption introduces a discontinuity in the contact load at the transition from elastic to elastic-plastic contact. This gave rise to several modifications to the original model, e.g. Evseev et al (1991) and Zhao et al (2000). Unfortunately, all these modifications are based on mathematical, rather than physical, considerations to smooth the discontinuity in the CEB model and do not provide a solution to the basic problem of lacking accuracy in the elastic-plastic contact regime. Such accurate solution calls for the use of a Finite Element Method (FEM). Kucharski et al (1994) used FEM to solve elastic-plastic contact of a single asperity and provided empirical expressions for the contact load and area. Surprisingly, the mean contact pressure in this analysis was, in some cases, higher than the indentation hardness and therefore unreasonable. Recently, Kogut \& Etsion (2002) presented an accurate elastic-plastic finite element solution for the contact of a deformable sphere pressed by a rigid flat. Their solution provides convenient dimensionless expressions for contact load and area covering a large range of interference from yielding inception to fully plastic contact of the sphere. The more accurate FEM solution (Kogut \& Etsion 2002) provides a solution to the basic problem in the intermediate elastic-plastic contact regime. More recently, Sellgren et al (2003) have presented a finite element-based model of contact between rough surfaces taking into account the properties of engineering surfaces.

In a recent study (Mukherjee et al 2004), the authors have presented an analysis of adhesion at the contact of rough surfaces offering a revision of the RG adhesion model of contacting rough surfaces by considering several regimes of deformation (elastic, elastic-plastic and fully plastic) of contacting asperities and uses the JKR model of contacts between elastic spheres and a rigid flat in the presence of adhesional surface forces. In that study only the Gaussian rough surfaces are considered and a recent short communication (Patra et al 2008) considers the asymmetric distribution of asperity heights. The present work extends the authors' earlier study (Mukherjee et al 2004) of elastic-plastic adhesive contact to non-Gaussian rough sliding surfaces and the Weibull distribution is adopted to represent surface roughness with 


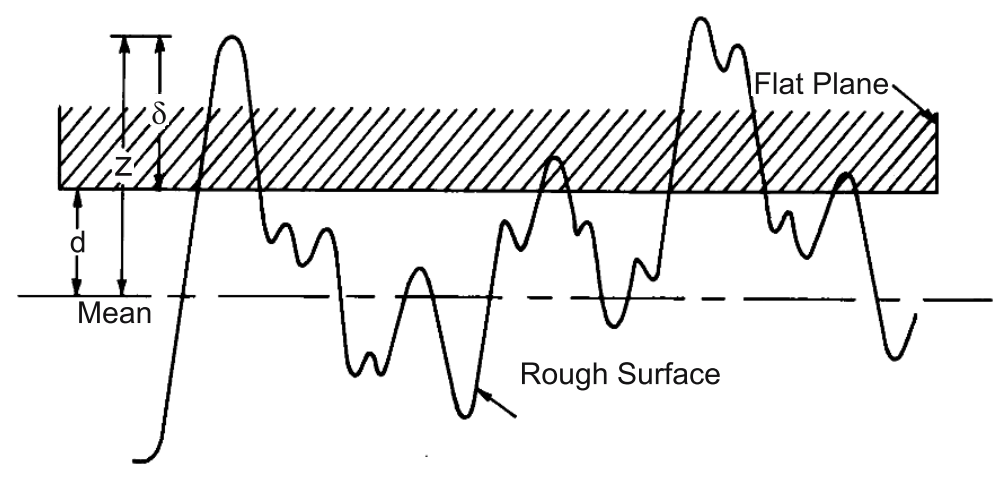

Figure 1. Contact between a rough deformable surface and a rigid flat plane.

asymmetric asperity heights. Thus the present study incorporates two new considerations in adhesive contact analysis of rough solids; the inclusion of elastic-plastic asperities along with purely elastic and purely plastic asperities, and an asymmetric distribution of asperity heights for the rough surfaces.

\section{Basic considerations}

\subsection{Rough surface contact model}

The contacting rough surfaces are modelled, in general, using Greenwood \& Williamson's (GW) statistical approach. Here it is assumed that when two rough surfaces contact with each other under the application of a normal load, actual contact occurs at the peak of the asperities of the rough surfaces. Due to the application of load the asperities will be deformed and the deformation may be elastic, plastic or elastic-plastic depending on contact pressure, material and surface properties. In such a model, the two rough surfaces in contact are represented by an equivalent rough surface in contact with a smooth plane as shown in figure 1 . The model assumes that the asperities are far apart, and there is no interaction between them and no bulk deformation occurs during contact. In this model asperities are assumed to have spherical cap of radius $R$ and their heights to follow Gaussian distribution with the probability density function given by

$$
\phi(z)=\frac{1}{\sigma \sqrt{2 \pi}} \exp \left[-\frac{z^{2}}{2 \sigma^{2}}\right],
$$

where $\sigma$ is the standard deviation and $z$ is the height of an asperity measured from the mean.

The main advantage of selecting Gaussian distribution is that this distribution is reproductive under addition, i.e. sum of Gaussian random variables is also Gaussian. Hence by using this distribution the contact of two rough surfaces can easily be represented by the contact of an equivalent rough surface having asperities with Gaussian height distribution and a smooth plane. But the major drawback of using this distribution is that being a symmetric one its skewness is zero and hence it cannot truly represent the skewness generated by finishing operations, e.g. grinding, polishing, lapping, etc. and other processes that produce asymmetric/skewed surface roughness. In such situation Weibull distribution is a more general one where skewness effect is easily included. Though Weibull distribution is not reproductive 
under addition it is possible to find an equivalent Weibull distribution that has the same mean and variance as the sum of any two Weibull random variables (McCool 2000). Also it has been shown (Kotwal \& Bhushan 1996) that two non-Gaussian surfaces in contact are equivalent to a flat surface in contact with a composite rough surface. In the present work both Gaussian and Weibull distributions have been used.

The Weibull distribution can take on the characteristics of other types of distributions, based on the value of the shape parameter, $\beta$. In the present work it is used to represent the distributions of asperity heights. The probability density function of a two-parameter Weibull distribution is given by

$$
\phi(z)=\frac{\beta z^{\beta-1}}{\eta^{\beta}} \exp \left[-(z / \eta)^{\beta}\right], z>0, \beta>0, \eta>0,
$$

where $\beta$ and $\eta$ are the shape and scale parameters respectively. The $n$-th moment of the Weibull distribution $M_{n}$ is defined as

$$
M_{n}=E\left(z^{n}\right)=\int_{0}^{\infty} z^{n} \phi(z) d z=\eta^{n} B_{n},
$$

where $B_{n}$ is the Gamma function given by

$$
B_{n}=\Gamma(1+n / \beta) \text {. }
$$

The mean (expectation) of this distribution is

$$
E(z)=\eta B_{1}=M_{1}
$$

and its variance is

$$
\sigma^{2}=M_{2}-M_{1}^{2}=\eta^{2}\left[B_{2}-B_{1}^{2}\right] .
$$

The skewness $(S k)$ and kurtosis $(K u)$ of Weibull distribution are given by

$$
\begin{aligned}
S k & =\frac{B_{3}-3 B_{2} B_{1}+2 B_{1}^{3}}{\left(B_{2}-B_{1}^{2}\right)^{3 / 2}} \\
\text { and } K u & =\frac{B_{4}-4 B_{3} B_{1}+6 B_{2} B_{1}^{2}-3 B_{1}^{4}}{\left(B_{2}-B_{1}^{2}\right)^{2}} .
\end{aligned}
$$

Variance, skewness and kurtosis can be extracted/measured from the surface roughness profile. From eqn. (4), (7) and (8) it is clearly seen that skewness and kurtosis are dependent only on shape parameter $(\beta)$. To calculate the Weibull parameters one can ignore anyone of $S k$ and $K u$. In the present work $K u$ has been ignored for simplicity and then eqn. (7) has been solved to get the value of the shape parameter $(\beta)$ corresponding to a particular value of $S k$. Then using this value of $\beta$ in eqn. (6) $\eta$ is obtained. To calculate the Weibull parameters, one can proceed in an alternative method by using $K u$ and ignoring $S k$ value. However, the merits of the earlier method over the alternative one have been elegantly discussed (Yu \& Polycarpou 2002) and in the present case, the earlier method is adopted. It has been found that when $\beta=3 \cdot 6, S k=0$, i.e. Weibull distribution can be taken as an approximation to Gaussian distribution. But when $S k$ is equal to zero, $K u$ is 2.72 , which is less than that of 
the Gaussian distribution where $K u$ is 3 . Hence Weibull distribution cannot truly represent the Gaussian distribution. $S k$ is positive when $\beta<3.6$ and negative when $\beta>3 \cdot 6$. The probability density function of the Weibull distribution given by eqn. (2) is dimensional, as it depends on the scale parameter $(\eta)$, which has the unit of length. Therefore the distribution is non-dimensionalised as

$$
\bar{z}=\frac{z-E(z)}{\sigma}=\frac{z-\eta B_{1}}{\sqrt{\eta^{2}\left(B_{2}-B_{1}^{2}\right)}} .
$$

Also by definition,

$$
\bar{\phi}(\bar{z}) d \bar{z}=\phi(z) d z
$$

which gives the normalized Weibull distribution as

$$
\bar{\phi}(\bar{z})=\beta\left(B_{1}+\bar{z} \sqrt{B_{2}-B_{1}^{2}}\right)^{\beta-1} \sqrt{B_{2}-B_{1}^{2}} \exp \left[-\left(B_{1}+\bar{z} \sqrt{B_{2}-B_{1}^{2}}\right)^{\beta}\right] .
$$

Thus $\bar{\phi}(\bar{z})$ is only a function of the dimensionless shape parameter, which determines the asymmetry of the distribution, and the dimensionless asperity height $\bar{z}$. The normalized Gaussian distribution of asperity heights is given by

$$
\bar{\phi}(\bar{z})=\frac{1}{\sqrt{2 \pi}} \exp \left[-\bar{z}^{2} / 2\right] .
$$

However, in case of Gaussian distribution, $\bar{z}=z / \sigma$.

\subsection{Loading analysis}

During loading, asperities may be deformed in elastic, elastic-plastic or plastic modes. Following the analysis of Johnson et al (1971) of contact between a smooth sphere and a flat in the presence of surface forces, the load on elastically deformed asperities and the load on a plastically deformed asperity may be obtained from the analysis of Roy Chowdhury \& Pollock (1981). The contact load of an asperity in elastic-plastic regime may be obtained from the analysis of Kogut \& Etsion (2002). Using all these results, total loading force has been presented in a recent study (Mukherjee et al 2004). For brevity, the detailed formulation is omitted here. Considering the contact between a rigid smooth surface and a rough deformable surface with a distribution $\phi(z)$ of asperity heights $z$ as shown in figure 1, the asperity deformation is $\delta=z-d$. If $N$ is the number of asperities per unit area of the rough surface, the total applied load on all the asperities in contact per unit area may be written non-dimensionally in terms of adhesion indices $\theta$ and $\lambda$ as (Mukherjee et al 2004)

$$
\begin{aligned}
\bar{P}_{l}= & \int_{\Delta_{0}}^{\Delta_{c 1}}\left(\Delta^{3 / 2}-\frac{4 \cdot 34}{\theta^{1 / 2}} \Delta^{3 / 4}\right) \bar{\phi}(\Delta) d \Delta \\
& +\frac{1 \cdot 03}{\Delta_{c 1}^{1 \cdot 425}}\left(\Delta_{c 1}^{3 / 2}-\frac{4 \cdot 34}{\theta^{1 / 2}} \Delta_{c 1}^{3 / 4}\right) \int_{\Delta_{c 1}}^{\Delta_{c 2}} \Delta^{1 \cdot 425} \bar{\phi}(\Delta) d \Delta \\
& +\frac{1 \cdot 40}{\Delta_{c 1}^{1 \cdot 263}}\left(\Delta_{c 1}^{3 / 2}-\frac{4 \cdot 34}{\theta^{1 / 2}} \Delta_{c 1}^{3 / 4}\right) \int_{\Delta_{c 2}}^{\Delta_{c 3}} \Delta^{1 \cdot 263} \bar{\phi}(\Delta) d \Delta \\
& +\int_{110 \Delta_{c}}^{\infty}\left(7 \cdot 3 \frac{\lambda^{1 / 4}}{\theta^{1 / 2}} \Delta-\frac{6 \cdot 28}{\theta}\right) \bar{\phi}(\Delta) d \Delta,
\end{aligned}
$$


where $\bar{P}_{l}=\frac{P_{l}}{K N R^{1 / 2} \sigma^{3 / 2}}, \Delta=\frac{\delta}{\sigma}, \Delta_{c}=\frac{\delta_{c}}{\sigma}, \Delta_{c 1}=\frac{\delta_{c 1}}{\sigma}, \Delta_{c 2}=\frac{\delta_{c 2}}{\sigma}, \Delta_{c 3}=\frac{\delta_{c 3}}{\sigma}, h=\frac{d}{\sigma}$ and $\bar{\phi}(\Delta)$ is the normalized asperity height distribution function in terms of normalized asperity deformation $(\Delta)$. For Gaussian distribution,

$$
\bar{\phi}(\Delta)=\frac{1}{\sqrt{2 \pi}} \exp \left\{-(h+\Delta)^{2} / 2\right\}
$$

and for Weibull distribution (Appendix A),

$$
\bar{\phi}(\Delta)=\beta(h+\Delta)^{\beta-1}\left(B_{2}-B_{1}^{2}\right)^{\beta / 2} \exp \left\{-\left[(h+\Delta) \sqrt{B_{2}-B_{1}^{2}}\right]^{\beta}\right\} .
$$

Here $\delta_{c}$ is the critical deformation of an asperity at yielding inception, i.e. for the transition from elastic to elastic-plastic deformation that takes place when mean contact pressure exceeds $0.6 H$ (Tabor 1951). For $\delta<\delta_{c}$ and for $\delta>110 \delta_{c}$ the contact is perfectly elastic and perfectly plastic, respectively. Following Kogut \& Etsion (2002), the total elastic-plastic regime extends over asperity interference values in the range $\delta_{c} \leq \delta \leq 110 \delta_{c}$. This region consists of two sub-regions with a transition at $\delta=6 \delta_{c}$. $\delta_{c 1}$ is the apparent critical deformation corresponding to real critical displacement $\delta_{c}$ while $\delta_{c 2}$ and $\delta_{c 3}$ are the apparent displacements corresponding to real displacements $6 \delta_{c}$ and $110 \delta_{c}$ respectively. $\Delta_{0}$ is the non-dimensional apparent displacement corresponding to actual displacement $\delta=0 . \Delta_{c 1}$ and $\Delta_{c}$ to be used in Eqn. (13) may be obtained from the following relations (Mukherjee et al 2004)

$$
\begin{aligned}
& \Delta_{c 1}^{3 / 4}-2 \cdot 19 \frac{\lambda^{1 / 4}}{\theta^{1 / 2}} \Delta_{c 1}^{1 / 4}-\frac{4 \cdot 34}{\theta^{1 / 2}} \geq 0 \\
& \text { and } \Delta_{c}=\Delta_{c 1}-\frac{2 \cdot 89}{\theta^{1 / 2}} \Delta_{c 1}^{1 / 4}
\end{aligned}
$$

$\Delta_{0}$ may be obtained from Eqn. (17) by substituting $\Delta_{c}=0$ with $\Delta_{c 1}=\Delta_{0}$ and written as

$$
\Delta_{0}=\frac{4 \cdot 125}{\theta^{2 / 3}}
$$

$\Delta_{c 2}$ and $\Delta_{c 3}$ to be used in Eqn. (13) may be obtained in a similar way from Eqn. (17) by replacing $\Delta_{c}$ by $6 \Delta_{c}$ with $\Delta_{c 1}=\Delta_{c 2}$ and $\Delta_{c}$ by $110 \Delta_{c}$ with $\Delta_{c 1}=\Delta_{c 3}$ respectively.

\subsection{Unloading and separation}

During unloading, contacting asperities separate in two possible modes-either a brittle mode or a ductile mode. Possibilities of both the separation modes have been discussed at length (Roy Chowdhury \& Ghosh 1994; Roy Chowdhury et al 1980). Considering both the separation modes of elastically deformed asperities, plastically deformed asperities and elastic-plastic asperities, the total unloading force for all the asperities in contact may be written in nondimensional form as (Mukherjee et al 2004)

$$
\begin{aligned}
\bar{P}_{u}= & -\int_{0}^{\frac{1}{\lambda}} \frac{7 \cdot 3 \lambda^{1 / 4}}{\theta^{1 / 2}} \Delta \bar{\phi}(\Delta) d \Delta+\int_{\frac{1}{\lambda}-\Delta_{b}}^{110 \Delta_{c}} \frac{4 \cdot 71}{\theta} F\left(\frac{\Delta}{\Delta_{b}}\right) \bar{\phi}(\Delta) d \Delta \\
& -\Delta_{e} \int_{L}^{\infty} 2 \cdot 12 \Delta^{1 / 2} \bar{\phi}(\Delta) d \Delta,
\end{aligned}
$$


where $\bar{P}_{u}=\frac{P_{u}}{K N R^{1 / 2} \sigma^{3 / 2}}, \Delta_{b}=\frac{\delta_{b}}{\sigma}, \Delta_{e}=\frac{\delta_{e}}{\sigma}, L$ is $\frac{1}{\lambda}, 110 \Delta_{c}$ or $\Delta_{e}^{*}$ whichever is the largest, and $\Delta_{e}^{*}=\frac{9 \theta^{2} \Delta_{e}^{4}}{128 \pi^{2}}$. The first term in right hand side of the Eqn. (19) represents the unloading force for asperities those extend in ductile mode, the second term represents the unloading force for brittle separation of elastic and elastic-plastic asperities and the third one represents the unloading force for brittle separation of plastically loaded asperities. The negative sign of the first and third integral indicates the tensile nature of the forces. The second integral is evaluated directly from the load displacement relation and hence indicates both the magnitude and sense of the force. Here $\delta_{e}$ is the elastic displacement of a plastically deformed asperity before separation. To take into account the history of loading, $\Delta_{e}$ is taken as $h-h_{0}$, where $h_{0}$ refers to the final value of the non-dimensional separation during loading. Here unloading force calculation must consider the release of applied compressive load before tensile load is applied. The unloading force in Eqn. (19) takes into account of this release of applied load as unloading is computed from the depth $\left(h_{0}\right)$ to which the flat was initially loaded. $F\left(\Delta / \Delta_{b}\right)$ is obtained by inverting Johnson's expression (1976) for $\left(\delta / \delta_{b}\right)$ where $\delta_{b}$ is the maximum extension of the tip of an asperity above its undeformed height before separation. Now using Eqn. (15) and (19), the unloading force for non-Gaussian surfaces in contact may be evaluated in terms of the adhesion indices and skewness values.

\section{Results and discussion}

Equations described in the previous sections are solved numerically to obtain the nondimensional loading and unloading force for different combinations of non-dimensional mean separation $h$, skewness values $S k$ and adhesion indices $\theta$ and $\lambda$. Before analysing the results it is important to consider the physical implications of the adhesion controlling parameters, $\theta$ and $\lambda$. The elastic and plastic adhesion indices $\theta$ and $\lambda$ merely indicate the relative importance of surface force induced adhesion for elastically and plastically deformed asperities as compared to the elastic and plastic forces on an individual asperity. Limiting values of $\theta$ and $\lambda$ are usually quoted as 10 and 0.125 respectively, beyond which effect of adhesion becomes insignificant due to surface roughness effect. In view of these limiting values, typical combinations of $\theta$ and $\lambda$ are considered in the present case in order to analyse the effect of elastic and plastic adhesion on loading - unloading behaviour of asymmetric rough surfaces. At this point, it may be noted that in a non-adhesive contact the nature of contact is indicated by the plasticity index $\left(\psi=\frac{E}{H} \sqrt{\frac{\sigma}{R}}\right)$ defined by Greenwood \& Williamson (1966). For $\psi<0 \cdot 6$, the contact is predominantly elastic and for $\psi>1 \cdot 0$, the contact is predominantly plastic. In an adhesive contact like the present one, the adhesion controlling parameters are $\theta$ and $\lambda$ and these may be related to the plasticity index, $\psi$ as $\psi=0 \cdot 65\left(\frac{\theta^{1 / 2}}{\lambda^{1 / 4}}\right)$. Table 1 shows typical combinations of $\theta$ and $\lambda$ considered in the present analysis and the corresponding $\psi$ values. It is seen that these combinations of $\theta$ and $\lambda$ cover the whole range of contact situations from predominantly elastic to predominantly plastic cases.

The skewness of a surface profile represents the asymmetric spread of the height distribution. A Gaussian surface has zero skewness with an equal number of peaks and valleys at certain heights. Profiles with peaks removed have negative skewness whereas profiles with valleys filled or high peaks have positive skewness. It has been shown (Kotwal \& Bhushan 1996) that for $S k>2$, the Weibull distribution becomes a one sided distribution on the contact side of the rough surface. For $S k<-2$, the Weibull parameters become very large and impractical. The most practical skewness values lie in the range of $-1<S k<+1$. For a symmetric Weibull distribution, i.e. $S k=0$, the kurtosis is $K u=2 \cdot 72$, which is less than 
Table 1. Adhesion indices and plasticity index.

\begin{tabular}{llllll}
\hline$\theta$ & $\lambda$ & $\psi$ & $\theta$ & $\lambda$ & $\psi$ \\
\hline $0 \cdot 1$ & $0 \cdot 05$ & 0.29 & 10 & 0.05 & 4.35 \\
& $0 \cdot 1$ & $0 \cdot 20$ & & $0 \cdot 1$ & 3.65 \\
& $0 \cdot 2$ & 0.15 & & $0 \cdot 2$ & $3 \cdot 07$ \\
2 & 0.05 & 1.95 & 20 & 0.05 & $6 \cdot 15$ \\
& $0 \cdot 1$ & 1.63 & & $0 \cdot 1$ & 2.90 \\
& $0 \cdot 2$ & 1.37 & & 0.2 & $2 \cdot 45$ \\
\hline
\end{tabular}

the true Gaussian distribution where $K u$ equals to 3. Thus there is some error in using the Weibull to approximate the Gaussian distribution. However for the present analysis, skewness values of the range $-1<S k<+2$ are considered. The non-dimensional mean separation ( $h \equiv d / \sigma$ ) is considered between 0 to +4 . At larger separations, $h>4$, the number of asperities in contact is very small, as evidenced from the magnitude of contact load in most of the cases. On the other hand, for very small separation, $h<0$, the present asperity based model may give erroneous results since asperities may undergo very large deformations, which may violate the basic assumption of no interaction between them.

Non-dimensional loading force against non-dimensional mean separation for different combinations of skewness values and elastic and plastic adhesion indices are shown in figure 2 . Figures 2(a) and 2(b) show the variation of loading force with mean separation for varying skewness values at $\theta=0 \cdot 1, \lambda=0.05$ and 0.2 . At these combinations, applied load is negative and magnitude of this load increases with decreasing mean separation. Negative applied load means tensile force indicating strong adhesion. Table 1 shows that for these combinations of $\theta$ and $\lambda$, the contact is predominantly elastic and low value of $\theta$ implies strong adhesion in elastically deformed asperities. This leads to negative applied load for such combinations. Comparison between figures 2(a) and 2(b) shows that the change in $\lambda$ value has insignificant effect on loading behaviour for such predominantly elastic contact situations. Figures 2(c)2(f) show the variation of applied load against mean separation for other combinations of $\theta$ and $\lambda(\theta=2,10$ and $\lambda=0 \cdot 05,0 \cdot 2)$ where contact is mostly plastic as seen from table 1 . The general trend of behaviour for these cases is that contact load is compressive indicating smaller adhesion effect. As the mean separation decreases, loading force increases due to more number of asperities coming in contact. For mean separation beyond zero, the contact load is almost zero except for negative skewness values indicating that the number of asperities in contact is very small. For negative skewness, however, loading force is significant even for positive mean separation. At a particular mean separation, the loading force is small at high skewness and monotonically increases with decrease in skewness values. Positive skewness indicates that the profile has high peaks and fewer numbers of asperities above the mean leading to smaller contact area. As a result, smaller amount of external applied force is needed for such cases in order to maintain a particular contact situation. At negative skewness the profiles have peaks removed and hence contact area is more at particular mean separation leading to a higher loading force. For comparison, the loading forces for Gaussian rough surfaces are evaluated for the same parametric combinations and included in the same plots. It is seen that for all typical combinations of the adhesion indices there is significant difference between loading force for Weibull distribution with $S k=0$ and the same for Gaussian distribution. Thus the symmetric Weibull distribution cannot truly represent a Gaussian dis- 


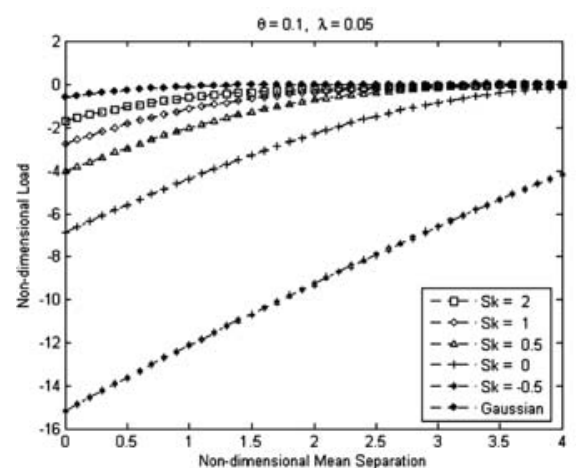

(a)

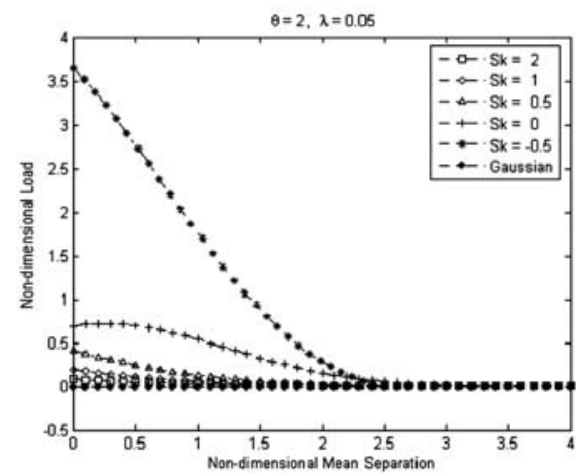

(c)

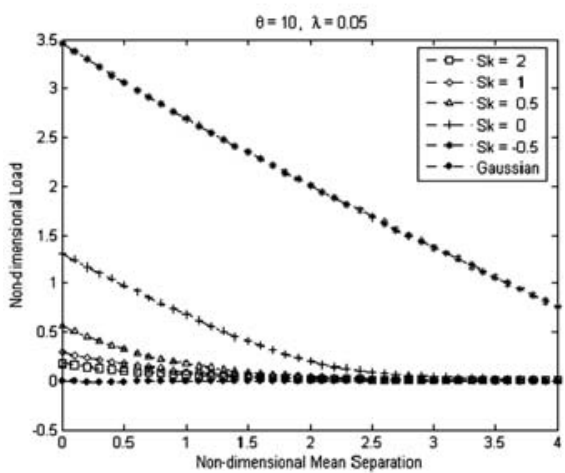

(e)

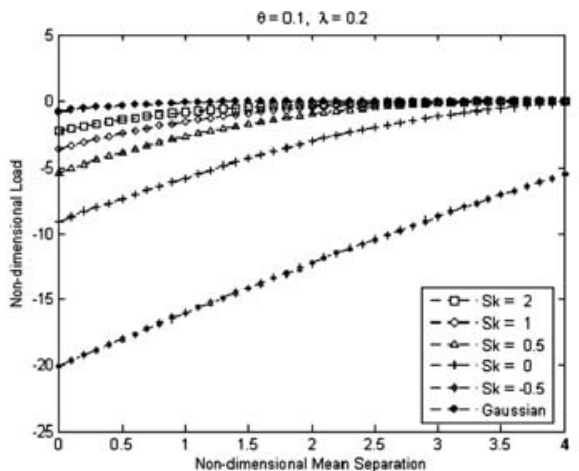

(b)

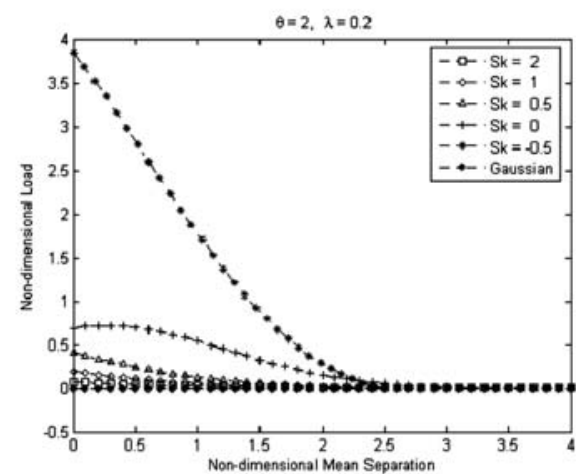

(d)

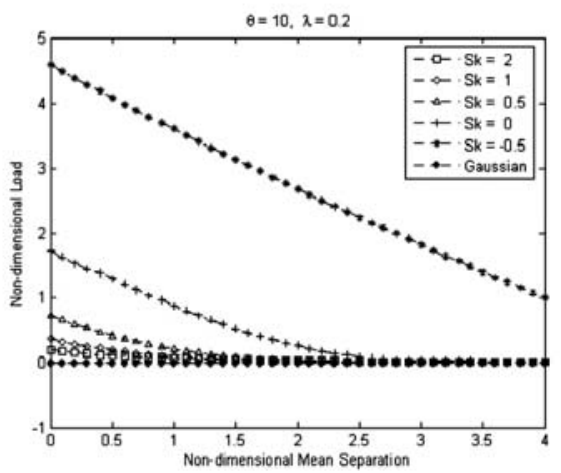

(f)

Figure 2. Plots of loading force against mean separation for varying $\theta, \lambda$ and skewness.

tribution. It may be noted here that in a non-adhesive contact situation, the Gaussian surface contact results act as a boundary between the results with positive skewness and the results with negative skewness (Yu and Polycarpou 2002). But in the present adhesive contact situation, a different behaviour is observed. The asymmetric surface contact results including both positive and negative skewness values differ significantly from the Gaussian results and all these lie on one side of the Gaussian results. This is probably due to combined effect of 


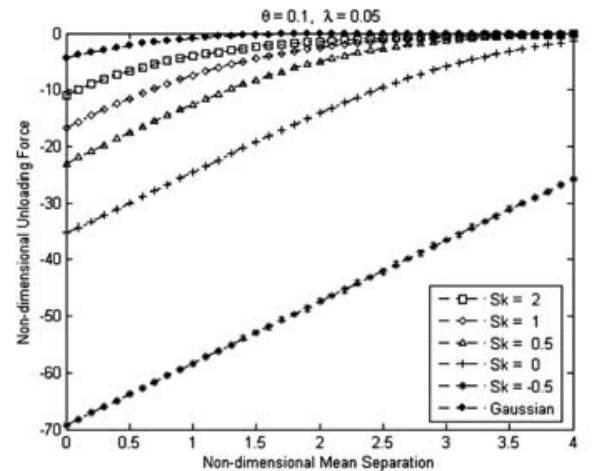

(a)

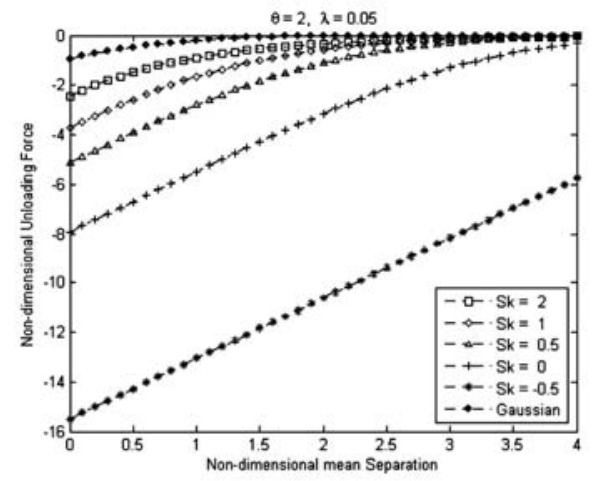

(c)

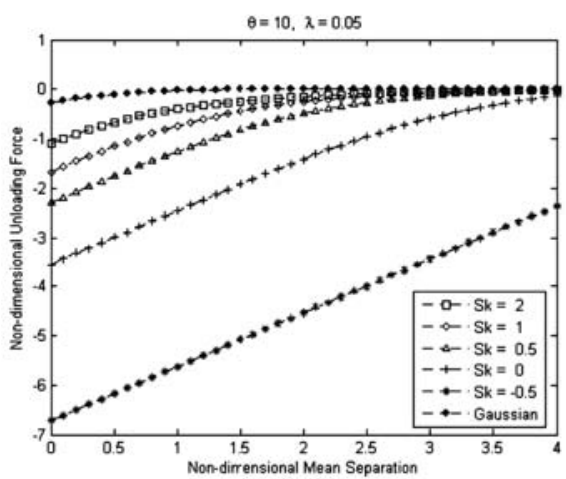

(e)

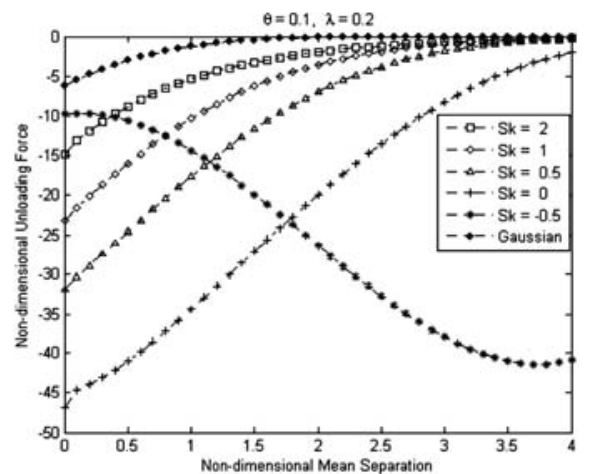

(b)

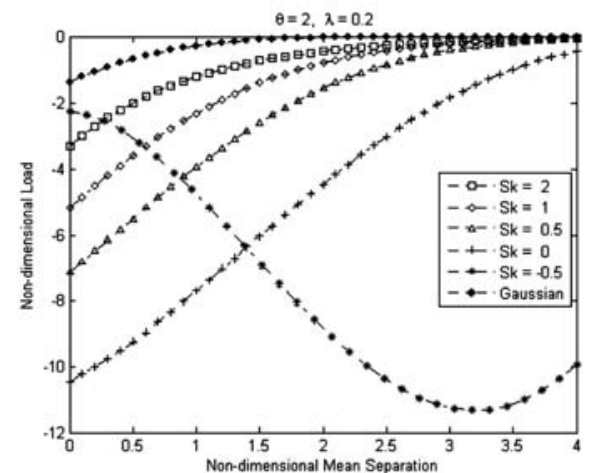

(d)

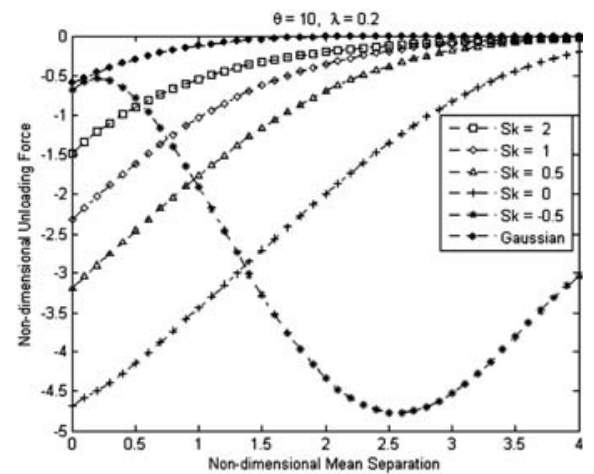

(f)

Figure 3. Plots of unloading force against mean separation for varying $\theta, \lambda$ and skewness.

adhesion and the asymmetric roughness features. It is seen from all these loading force plots that the loading behaviour is influenced by both elastic and plastic adhesion indices. Also the skewness value largely influences the loading characteristics in presence of adhesion.

Figure 3 shows the plots of non-dimensional unloading force against non-dimensional mean separation for similar combinations of elastic and plastic adhesion indices as considered for loading analysis. It may be noted that the surfaces unload from the final loaded position and the 
unloading force results are strongly dependent on the mean separation where unloading starts from. During loading analysis, the non-dimensional mean separation $(h \equiv d / \sigma)$ is considered from +4 to 0 . Thus, the unloading starts at zero mean separation. The unloading force results presented here are the results of unloading from a situation where non-dimensional mean separation equals zero, i.e. $h_{0}=0$. The maximum negative value of the unloading force gives the total pull off force of a surface whose asperities may have deformed in elastic and plastic mode and separate in either ductile or brittle manner. As it can be seen, during separation the non-dimensional unloading force gradually falls to zero as the higher asperities finally separate. In general, the tensile unloading force is more for smaller skewness values at a particular mean separation. At a particular combination, e.g. at $\theta=2$ and $\lambda=0 \cdot 2$, the maximum unloading force (tensile) is almost same for different skewness values but their location along the mean separation axis varies. At negative skewness, it occurs at positive mean separation and shifts towards negative mean separation as the skewness value is gradually increased. However, the location of the maximum unloading force is also influenced by the combination of adhesion indices, $\theta$ and $\lambda$. For same parametric combinations the unloading force for Gaussian rough surfaces is also included in these plots and it can be seen that at a particular mean separation, the unloading force is, in general, least for Gaussian surfaces. Also there is significant difference between unloading force for Weibull distribution with $S k=0$ and the same for Gaussian distribution. For all combinations of $\theta$ and $\lambda$, the general trend of behaviour remains the same but the magnitude of unloading force at a particular mean separation vary with combinations of adhesion indices. The unloading behaviour is seen to depend on both elastic and plastic adhesion indices and the skewness value largely influence the unloading characteristics of non-Gaussian rough surfaces in presence of adhesion.

Pull-off forces derived from these unloading results are plotted against elastic adhesion indices for different plastic adhesion indices and at two particular skewness values are shown in figures 4(a) and 4(b). A change in slope may be detected at certain values of $\theta$ indicating low adhesion beyond these values. This critical value of $\theta$ is dependent on $\lambda$ and it is seen that critical $\theta$ of 10 as cited in Fuller and Tabor's work (1975) is a special case in the generalised parametric variation given in figures 4(a) and 4(b). Similarly, in figures 4(c) and 4(d), pull-off forces are plotted against plastic adhesion indices $(\lambda)$ for different elastic adhesion indices $(\theta)$ and at two particular skewness values. The pull-off force is found to be maximum in the range of $\lambda$ values $0.075-0.25$ depending on $\theta$ and $S k$ combination. Thus in general no critical value of $\lambda$ is predicted beyond which adhesion may be considered to be low. It indicates that critical $\lambda$ value of 0.125 indicating low adhesion is not universal and it also represents a special case in the generalised parametric variation given in figures 4(c) and 4(d). In figures 4(e) and 4(f), pull-off forces are plotted against skewness values for different plastic adhesion indices $(\lambda)$ and at two typical values of elastic adhesion indices $(\theta)$. In general, pull-off force is more at negative skewness values indicating large adhesion for such surfaces. At this point, it may be noted that polycrystalline silicon MEMS surfaces with positive skewness exhibit low adhesion compared to surfaces with negative skewness (Komvopoulos 1996). This is in agreement with the present results. Thus for situations requiring low adhesion, the surface and material parameters may be chosen so as to yield high $\theta$, high positive skewness and the $\lambda$ value either very low or very high for asymmetric rough surfaces.

In order to facilitate a comparison between the RG model (1994) and the present improved model, total loading and unloading forces for the two models are plotted for typical combinations of adhesion indices and skewness values in figure 5. As can be seen from figures 5(a) and 5(b), the loading force from the present analysis significantly differs from the RG model (1994) and the amount of difference is dependent on $\theta$ and $\lambda$ combination. In general, the 


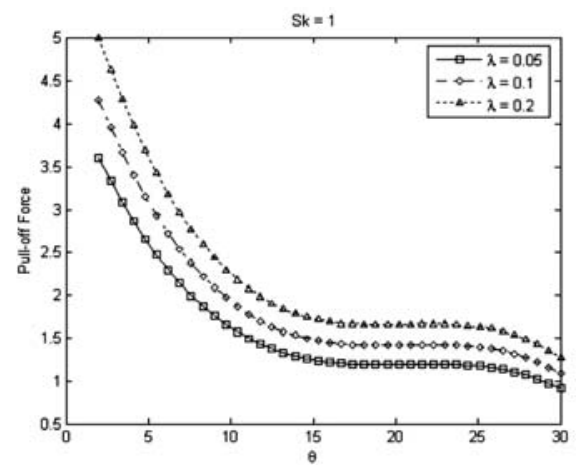

(a)

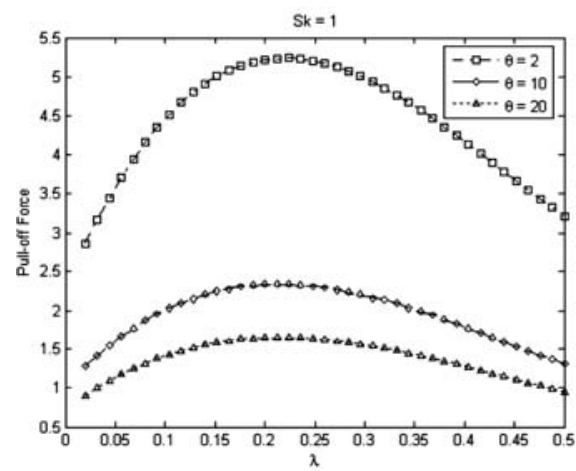

(c)

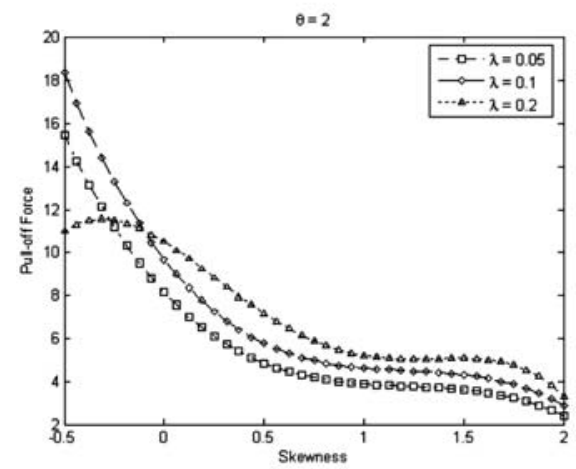

(e)

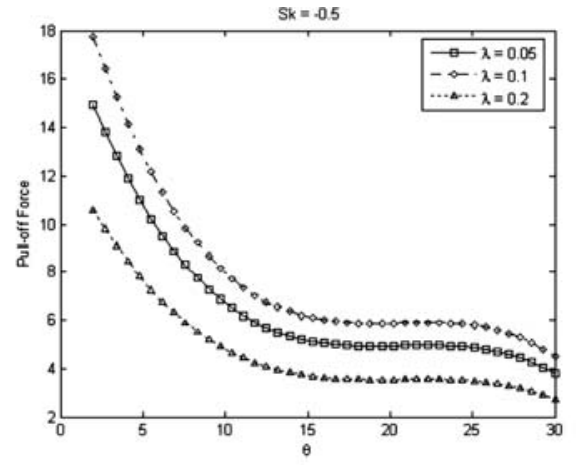

(b)

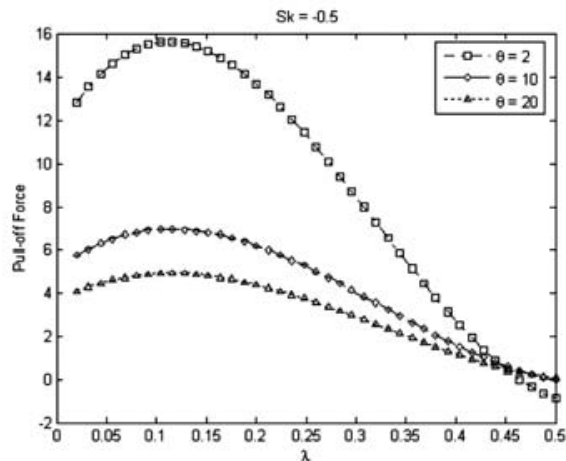

(d)

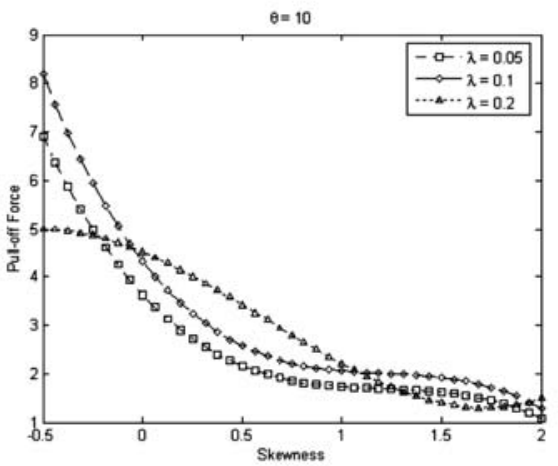

(f)

Figure 4. Variation of pull-off force as functions of $\theta, \lambda$ and skewness.

loading force for present analysis at smaller mean separations is relatively less as compared to the RG model. Also it may be noted that the difference in loading force from the two models is comparatively higher for negative skewness values. As can be seen from figures 5(c) and 5(d), the unloading forces from the two analyses are almost same. Thus a comparison with RG model (1994) shows substantial differences in the loading force, but insignificant difference in the unloading force. 


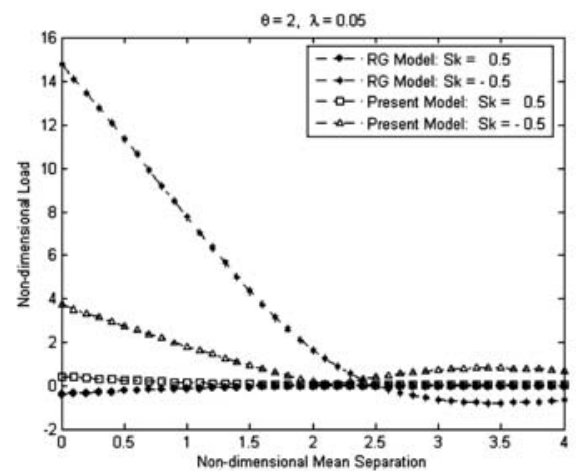

(a)

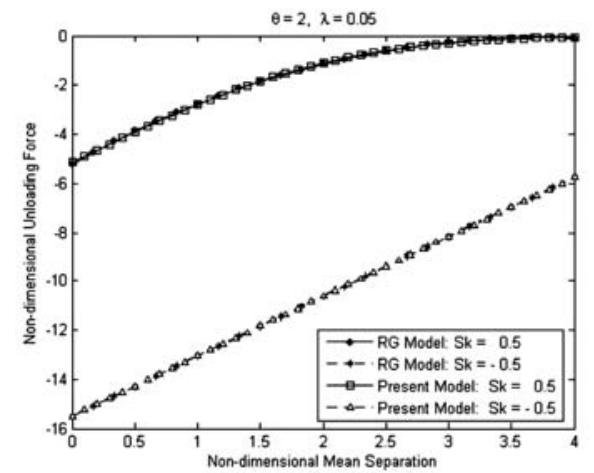

(c)

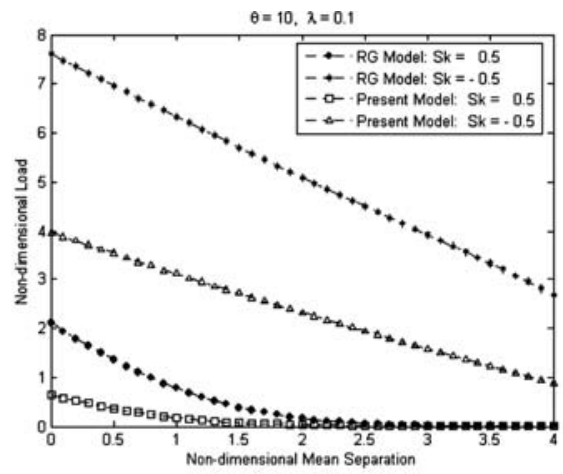

(b)

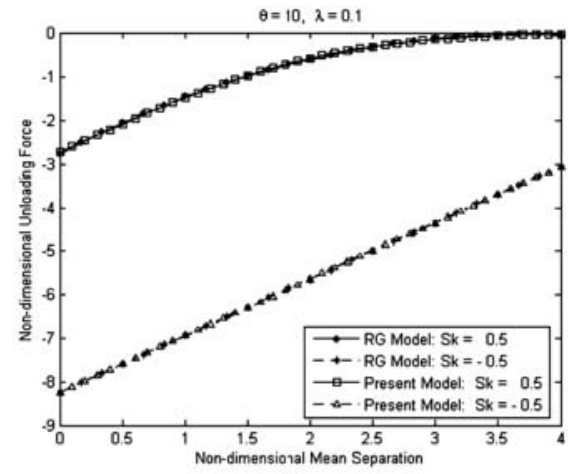

(d)

Figure 5. Loading and unloading forces against mean separation - A comparison between present model and the RG model.

\section{Conclusions}

An improved elastic-plastic model for the adhesive contact of rough surfaces with asymmetric asperity height distribution is presented. The Weibull distribution is used to model the asymmetry with skewness as the key parameter. In addition to fully elastic and fully plastic regime, considered in earlier analyses, the present model considers the intermediate elasticplastic regime based on an accurate FEM solution of a single asperity contact. Therefore, the present model represents a more realistic situation than previous approximate ones. A comparison with one such approximate model shows substantial differences in the loading force depending on combinations of non-dimensional mean separation, skewness values and adhesion indices. The loading and unloading behaviour is conveniently described in terms of $\theta$ and $\lambda$, and skewness. It is found that loading-unloading behaviour is strongly influenced by adhesion indices and skewness values. Critical values of adhesion indices and skewness at which the effect of surface forces become insignificant are found to depend on material and surface parameters and they have been obtained for a range of parametric variation. For situations requiring low adhesion, the surface and material properties may be selected such as to yield high $\theta$, high positive skewness and the $\lambda$ value either very low or very high for asymmetric rough surfaces. 


\section{Appendix A}

The probability distribution function of Weibull distribution is

$$
\phi(z)=\frac{\beta z^{\beta-1}}{\eta^{\beta}} \exp \left[-(z / \eta)^{\beta}\right]
$$

By definition

$$
\phi(z) d z=\bar{\phi}(\bar{z}) d \bar{z},
$$

where

$$
\bar{z}=\frac{z-E(z)}{\sigma}=\frac{z-\eta B_{1}}{\sqrt{\eta^{2}\left(B_{2}-B_{1}^{2}\right)}}
$$

Which gives

$$
z=\eta \sqrt{B_{2}-B_{1}^{2}} \bar{z}+\eta B_{1}
$$

Again,

$$
\bar{z}=\frac{z-E(z)}{\sigma}=\frac{\sigma(h+\Delta)-\eta B_{1}}{\sigma}=h+\Delta-\frac{B_{1}}{\sqrt{B_{2}-B_{1}^{2}}}
$$

and $d \bar{z}=d \Delta$

From (A2),

$$
\begin{aligned}
\bar{\phi}(\bar{z}) d \bar{z}= & \phi(z) d z \\
= & \frac{\beta}{\eta^{\beta}} \eta^{\beta-1}\left(B_{1}+\bar{z} \sqrt{B_{2}-B_{1}^{2}}\right)^{\beta-1} \\
& \times \exp \left[-\left(B_{1}+\bar{z} \sqrt{B_{2}-B_{1}^{2}}\right)^{\beta} \eta \sqrt{B_{2}-B_{1}^{2}} d \bar{z}\right] .
\end{aligned}
$$

Thus

$$
\begin{aligned}
\bar{\phi}(\Delta)= & \beta\left[B_{1}+\sqrt{B_{2}-B_{1}^{2}}\left(h+\Delta-\frac{B_{1}}{\sqrt{B_{2}-B_{1}^{2}}}\right)\right]^{\beta-1} \sqrt{B_{2}-B_{1}^{2}} \\
& \cdot \exp \left\{-\left[B_{1}+\sqrt{B_{2}-B_{1}^{2}}\left(h+\Delta-\frac{B_{1}}{\sqrt{B_{2}-B_{1}^{2}}}\right)\right]^{\beta}\right\} \\
= & \beta(h+\Delta)^{\beta-1}\left(B_{2}-B_{1}^{2}\right)^{\beta / 2} \exp \left\{-\left[(h+\Delta) \sqrt{B_{2}-B_{1}^{2}}\right]^{\beta}\right\} .
\end{aligned}
$$




\section{Notation}

$d \quad$ mean separation of the surfaces

$E_{1}, E_{2} \quad$ elastic moduli of contacting materials

$E=\left[\left(1-v_{1}^{2}\right) / E_{1}+\left(1-v_{2}^{2}\right) / E_{2}\right]^{-1}$

$E(z)$ mean or expectation of Weibull distribution

$h \quad d / \sigma$

$h_{0} \quad$ minimum value of non-dimensional mean separation, $h$

$H \quad$ hardness

$K=4 E / 3$

$K u \quad$ kurtosis

$M_{n} \quad n$-th moment of the Weibull distribution

$N \quad$ number of asperities per unit area

$P_{l} \quad$ total loading force

$P_{u} \quad$ total unloading force

$R \quad$ asperity radius

Sk skewness

$z \quad$ height of an asperity

$\beta \quad$ shape parameter in Weibull distribution

$\gamma \quad$ work of adhesion per unit area

$\eta \quad$ scale parameter in Weibull distribution

$\delta \quad$ asperity deformation

$\delta_{b} \quad$ critical deformation of asperity for brittle separation

$\delta_{c} \quad$ critical displacement of an asperity for yielding inception

$\delta_{c 1} \quad$ apparent critical displacement of an asperity for yielding inception

$\delta_{c 2} \quad$ apparent displacement of an asperity corresponding to a real displacement of $6 \delta_{c}$

$\delta_{c 3} \quad$ apparent displacement of an asperity corresponding to a real displacement of $110 \delta_{c}$

$\delta_{e} \quad$ elastic displacement of a plastically deformed asperity before separation

$\begin{array}{ll}\Delta & \delta / \sigma \\ \Delta_{b} & \delta_{b} / \sigma \\ \Delta_{c} & \delta_{c} / \sigma \\ \Delta_{c_{1}} & \delta_{c_{1}} / \sigma \\ \Delta_{c_{2}} & \delta_{c_{2}} / \sigma \\ \Delta_{c_{3}} & \delta_{c_{3}} / \sigma \\ \Delta_{e} & \delta_{e} / \sigma \\ \Delta_{e}^{*} & \frac{9 \theta^{2} \Delta_{e}^{4}}{128 \pi^{2}}\end{array}$

$\Delta_{o} \quad$ non-dimensional apparent displacement of an asperity corresponding to zero real displacement

$\theta \quad$ elastic adhesion index $\left[\theta=K \sigma^{3 / 2} R^{1 / 2} /(\gamma R)\right]$

$\lambda \quad$ plastic adhesion index $\left[\lambda=\pi^{2} R H^{4} \sigma /\left(18 K^{2} \gamma^{2}\right)\right]$

$\psi \quad$ plasticity index $\left[\psi=\frac{E}{H} \sqrt{\frac{\sigma}{R}}\right]$

$v_{1}, v_{2} \quad$ Poisson's ratios of contacting materials

$\sigma \quad$ standard deviation of surface roughness

$\phi(z) \quad$ probability density function for asperity height distribution

\section{Superscript}

- non-dimensional form 


\section{References}

Baumgart P, Krajnovih D J, Nguyen T A, Tam A C 1995 A new laser texturing technique for high performance magnetic disk drives. IEEE Trans. Magn. 31: 2946-2951.

Bhushan B 1999 Handbook of micro/nanotribology. Boca Raton: CRC Press

Bowden F P, Rowe G W 1956 The adhesion of clean metal. Proc. Roy. Soc. (Lond.) A233: 429-442

Chang W R, Etsion I, Bogy D B 1987 An elastic-plastic model for the contact of rough surfaces Trans. ASME: J. Trib. 109: 257-263

Chang W R, Etsion I, Bogy D B 1988 Adhesion model for metallic rough surfaces. Trans. ASME: J. Trib. 110: 50-56

Derjaguin B V, Muller V M, Toporov Y P 1975 Effect of contact deformation on the adhesion of elastic solids. J. Coll. Interface Sci. 53: 314-326s

Evseev D G, Medvedev B M, Grigoriyan G G 1991 Modification of the elastic-plastic model for the contact of rough surfaces. Wear 150: 79-88

Fuller K N G, Tabor D 1975 The effect of surface roughness on the adhesion of elastic solids. Proc. Roy. Soc. (Lond.) A345: 327-342

Greenwood J A, Williamson J B P 1966 Contact of nominally flat surfaces. Proc. Roy. Soc. (Lond.) A295: 300-319

Johnson K L, Kendall K, Roberts A D 1971 Surface energy and the contact of elastic solids. Proc. Roy. Soc. (Lond.) A324: 301-313

Johnson K L 1976 Adhesion at the contact of solids, in: W T Koiter (ed.), Theoretical and applied mechanics, North Holland, Amsterdam, pp. 133-143

Kogut L, Etsion I 2002 Elastic-plastic contact analysis of a sphere and a rigid flat. Trans. ASME: J. Appl. Mech. 69: 657-662

Komvopoulos K 1996 Surface engineering and microtribology for MEMS. Wear 200: 305-327

Kotwal C A, Bhushan B 1996 Contact analysis of non-Gaussian surfaces for minimum static and kinetic friction and wear. Trib. Trans. 39: 890-898

Kucharski S, Klimczak T, Polijaniuk A, Kaczmarek J 1994 Finite elements model for the contact of rough surfaces. Wear 177: 1-13

McCool J I 1992 Non-Gaussian effects in microcontact. Intl. J. Mach. Tools. Manuf. 32: 115-123

McCool J I 2000 Extending the capability of the Greenwood Williamson microcontact model. Trans. ASME: J. Trib. 122: 496-502

Mirzamaani M, Doerner M F 1996 Magnetic performance and tribology of sputter-textured thin film disks. IEEE Trans. Magn. 32: 3638-3641

Mukherjee S, Ali S M, Sahoo P 2004 An improved elastic-plastic contact model of rough surfaces in the presence of adhesion. Proc. Instn. Mech. Engrs., Part J: J. Engg. Trib. 218: 557-567

Muller V M, Yushchenko V S, Derjaguin B V 1980 On the influence of molecular forces on the deformation of an elastic sphere and its sticking to rigid plane. J. Coll. Interface Sci. 77: 91-101

Patra S, Ali S M, Sahoo P 2008 Elastic-plastic adhesive contact of rough surfaces with asymmetric distribution of asperity heights. Wear 265(3-4): 554-559.

Ranjan R, Lambeth D N, Tromel M, Goglia P, Li Y 1991 Laser texturing for low-flying-height media. J. Appl. Phys. 69: 5745-5747

Roy Chowdhury S K, Ghosh P 1994 Adhesion and adhesional friction at the contact between solids. Wear 174: 9-19

Roy Chowdhury S K, Hartley N E W, Pollock H M, Wilkins M A 1980 Adhesion energies at a metal interface: the effects of surface treatments an ion implantation. J. Phys. D: Appl. Phys. 13: 17611784

Roy Chowdhury S K, Pollock H M 1981 Adhesion between metal surfaces: the effect of surface roughness. Wear 66: 307-321

Sellgren U, Bjorklund S, Andersson S 2003 A finite element-based model of normal contact between rough surfaces. Wear 254: 1180-1188

Stout K J, Davies E J, Sullivan P J 1990 Atlas of machined surfaces, London: Chapman and Hall 
Tabor D 1951 The hardness of metals, Oxford, UK: Oxford University Press

Thomas T R, Sayles R S 1978 Some problems in the tribology of rough surfaces. Trib. Intl. 11(3): $163-168$

Thomas T R 1982 Rough Surfaces, New York: Longman

Whitehouse D J, Archard J F 1970 The properties of random surfaces of significance in their contact. Proc. Roy. Soc. (Lond.) A316: 97-121

Whitehouse D J 1994 Handbook of Surface Metrology, Bristol, UK: Institute of Physics Publishing

Williamson J B P 1968 Topography of solid surfaces, in: P M Ku (ed.), Interdisciplinary approach to friction and wear, Washington (DC): SP - 181, NASA Special Publishing, NASA; pp. 85-142

Yu N, Polycarpou A A 2002 Contact of rough surfaces with asymmetric distribution of asperity heights. Trans. ASME: J. Trib. 124: 367-376

Zhao Y, Maietta D M, Chang L 2000 An asperity micro contact model incorporating the transition from elastic deformation to fully plastic flow. Trans. ASME: J. Trib. 122: 86-93 